\title{
Potenziale der Ablaufsimulation für die Entwicklung von einer Pilot- zur Volumenfertigung am Beispiel der Heliatek GmbH
}

\author{
Felix Diener ${ }^{1 *}$, Samuel Horler ${ }^{2}$, Pierre Grzona², Philipp Wilsky ${ }^{2}$ \\ ${ }^{1}$ Heliatek GmbH, Treidlerstraße 3, 01139 Dresden, *felix.diener@heliatek.com \\ 2Institut für Betriebswissenschaften und Fabriksysteme, Professur Fabrikplanung und Fabrikbetrieb, Technische \\ Universität Chemnitz, Erfenschlager Str. 73, 09125 Chemnitz, Deutschland
}

\begin{abstract}
In this paper the potential of an agile simulation proceeding in start-ups is described. The authors from the Chemnitz University of Technology worked together with Heliatek $\mathrm{GmbH}$ on the vision of a large scale organic solar film production. To accomplish the project goals, it was necessary to implement an agile simulation process, so that the right information could be gathered and integrated in the simulation environment. Due to this a high knowledge gain in the company and by the project participants could be reached, but also deficits of actual use of simulation tools in SME became visible. At the end, the knowledge gain could be transferred in specific guidance points for SME.
\end{abstract}

\section{Einleitung}

Die Simulation in Produktion und Logistik als Werkzeug der digitalen Fabrik hat sich in vielen Anwendungskontexten bewährt [1]. Gerade für Start-ups sowie kleine und mittlere Unternehmen (KMU) ergeben sich neben den vielfach versprochenen Chancen jedoch auch Hürden für die erfolgreiche Anwendung der Simulation in der Produktion. Insbesondere der Aufwand und das benötigte Wissen zur Erstellung einer Simulationsstudie in kleinen und mittleren Unternehmen bzw. Start-ups stellt eine große Hürde dar. Spezifisches Prozesswissen ist vorhanden, doch fehlt es meist an methodischem Wissen zum Einsatz und Grenzen von Simulationswerkzeugen für die Fabrikplanung.

Ergänzend benennt eine Studie des Bundesministeriums für Wirtschaft und Energie wirtschaftliche Potenziale von Simulation beim Einsatz im Mittelstand. Zusätzlich werden aber auch Hemmnisse bei einmaligen und laufenden Kosten von Simulationsumgebungen und deren Komplexität, Modularität und Nutzerfreundlichkeit gesehen. [2]

\section{Simulation in Start-Ups und KMU}

Beispiele für Einsatzmöglichkeiten von Simulation in KMU sind die durchgehende Planungsbegleitung und operative Produktionsplanung [3, 4]. Die Hürden konnten seit diesen Beiträgen im Vergleich zur aktuelleren Studie des BMWi gesenkt werden, insbesondere durch die angemahnte stärkere Einbindung in die Lehre [5]. Ein weiteres aktuelles Beispiel aus dem Jahr 2019 ist die Lern-App PSIMA, die sich mit der Gamification beim Erlernen des Umganges mit der Simulationsumgebung Tecnomatix Plant Simulation beschäftigt [6].

Ein weiterer Baustein ist der Ansatz über webbasierte Simulationsangebote die Investitionshürde für KMU zu senken, beispielsweise über das Projekt simKMU [7]. Eine Untersuchung aus dem Jahr 2014 kam zu dem Ergebnis, das kein Werkzeug am Markt die Anforderungen in Form eines KMU-gerechten Bausteinkasten erfüllt [8].

Aus der Literatur wird das enorme Potenzial von Simulation deutlich, aber auch, dass noch große Lücken bei der Durchdringung bei KMU und dementsprechend bei Start-ups als Teil dieser Gruppe vorherrschen. Die Erstellung von Simulationsstudien im Rahmen von Studienabschlussarbeiten ist hierbei ein probates Mittel, um den Aufwand für KMU zu reduzieren und strategisch Simulationswissen im Unternehmen aufzubauen. 


\section{Ausgangssituation bei der Heliatek GmbH}

Die Heliatek GmbH ist ein Hersteller von innovativen organischen Dünnschicht-Solarlösungen. $\mathrm{Zu}$ den Produkten zählen flexible, gebrauchsfertige Solarmodule für die Bauindustrie. Die weltweit erste qualitativ hochwertige Vakuum-Roll-to-Roll-Produktion garantiert die versprochenen Moduleigenschaften, stellt jedoch besondere Anforderungen an die Produktionsgestaltung.

Derzeit werden umfangreiche Pläne für die Erweiterung des Produktspektrums und hin zu einer Skalierung der Produktion umgesetzt. Die in der Start-up-Phase des Unternehmens realisierte Pilot-Fertigung soll deswegen um eine Volumenproduktion ergänzt werden. Die Planung und Umsetzung dieses neuen Produktionsbereiches sind aufgrund des Neuheitsgrades der Produkte und damit einhergehender Anlagenkomplexität sowie des starken Wachstums innerhalb des Unternehmens herausfordernd. Da keine vergleichbaren Best Practices existieren, können wichtige Erfahrungen häufig erst während der Planung gemacht werden. Um die Materialflüsse im neuen Produktionsbereich vorzudenken und zu optimieren, wurde eine Simulation angestrebt. Dabei wurden folgende Ziele verfolgt:

- Analyse der Transportmittel- und Anlagenauslastungen

- Aufzeigen von möglichen Engpässen

- Dimensionierung von Transportmitteln

- Visualisierung der Prozessabläufe

Entsprechend dieser Ziele, wurde gemeinsam mit der TU Chemnitz seit 2018 eine simulative Betrachtung durchgeführt und gleichzeitig untersucht, welche ergänzenden Potenziale mit dem Einsatz des Werkzeuges in einem stark wachsenden Unternehmensumfeld gehoben werden können.

So sollte neben der Erreichung der definierten Simulationsziele überprüft werden, welchen Nutzen, welche Chancen aber auch welche etwaigen Grenzen die Simulation speziell für Start-ups und KMU besitzt. Zu diesem Zweck wurde entschieden, über eine Befragung von Angestellten der Heliatek GmbH Aussagen zu diesen Fragestellungen zu erhalten. Im Nachgang wurden die Erwartungen der Beteiligten vor der Studie mit den tatsächlich generierten Ergebnissen abgeglichen.
Hierzu wurde ein Fragebogen entworfen und von ausgewählten Angestellten ausgefüllt. Die zentralen Fragestellungen adressierten folgende Punkte:

- Anforderungen/Wünsche/Erwartungen/Ziele an die Studie zur Simulation des Materialflusses

- konkrete Fragestellungen zu denen ein Erkenntnisgewinn erwartet wird

- Grenzen der Simulation bei der Heliatek $\mathrm{GmbH}$

- Abstraktion vom konkreten Anwendungsfall der Heliatek GmbH auf allgemeine Chancen und Probleme/Grenzen bei Start-Ups

Im Folgenden werden die Ergebnisse der Befragung zusammengefasst. Generell sollte ein Wissensaufbau zu den erwarteten Materialflüssen durch deren Analyse stattfinden, und es sollten greifbare Ergebnisse aus dem dynamischen Modell generiert werden. Die Anforderungen und Erwartungen an die Simulation decken sich zum großen Teil mit den Zielen und werden detailliert im Rahmen der Phase 1 der gewählten Vorgehensweise erläutert.

Im Anschluss an die Fragestellungen sollten die Probanden einschätzen, wo aus ihrer Sicht Grenzen der Simulationsstudie für die Heliatek $\mathrm{GmbH}$ sind. Hier wurde der Fakt herausgestellt, dass es sich um eine in dieser Größenordnung komplett neue Produktionsstrecke handelt und keine Datenbasis oder Erfahrungswerte zur Verfügung standen und so viele Inputdaten auf Annahmen basierten und eventuell nicht die Realität abbilden. Als weiterer Punkt wurde hier der begrenzte Detaillierungsgrad benannt, durch den die hochkomplexen Prozesse sowie die einzelnen Entstehungsstufen des Produkts nur schwer abzubilden waren.

Ausgehend von den getätigten Aussagen bezüglich der konkreten Simulationsstudie für das Unternehmen, wurden die Befragten anschließend gebeten zu abstrahieren, welche Möglichkeiten und auch Probleme sie generell beim Einsatz von Simulation in Start-ups und KMU sehen. Bezüglich der Möglichkeiten wurde die bessere Planbarkeit von Logistik und Produktion sowie die Beseitigung von Unsicherheiten im Vorfeld des Aufbaus einer neuen Produktionsstrecke erwähnt. Außerdem wurde hier die Erleichterung bei der Erarbeitung von Materialflusskonzepten sowie die Möglichkeit einer umfassenden Prozessanalyse genannt. 
Bei der Einschätzung von Grenzen und Problemen für Start-ups und KMU wurden die hohen Kosten für den Erwerb der Simulationssoftware und die erforderlichen Lizenzen genannt. Des Weiteren haben die Befragten den hohen zeitlichen Aufwand und das eventuell nicht vorhandene Know-how zur Erstellung von Simulationsmodellen angeführt. Zuletzt wurde noch das fehlende Verständnis zum Nutzen von Simulationsanwendungen für KMU angemerkt.

\section{Vorgehensweise bei der Simulations- studie bei der Heliatek $\mathrm{GmbH}$}

Nachdem der Einsatz der Ablaufsimulation in KMU ausgehend von Wissenschaft und Praxis am Beispiel der Heliatek GmbH beleuchtet wurde, kann resümiert werden, dass deren Einsatz unter gegeben Bedingungen erschwert ist. Aus diesem Grund wurde für die Simulationsstudie ein eigens konzipiertes Vorgehenskonzept ausgearbeitet und zur Anwendung gebracht.

Der nachfolgenden Ausführungen beschreiben das Vorgehen, die Herausforderungen und Ergebnisse der damit durchgeführten Simulationsstudie. Dabei werden insbesondere die aus der vorherrschenden Situation resultierenden Hürden beschrieben sowie transparent dargelegt, welchen Beitrag die Simulation im Planungsprozess des neuen Fertigungsbereiches leisten konnte. Der Beitrag adressiert also zudem den Nutzen der Simulationsstudie, welcher über die unmittelbaren Simulationsergebnisse hinausgeht und arbeitet weiterhin Handlungsempfehlungen für vergleichbare Problemstellungen heraus.

Das Vorgehen wurde in Anlehnung an das bewährte Simulationsvorgehensmodell nach VDI 3633 gewählt [9]. Basierend auf diesem Rahmen, wurden besonders die Schritte der Zielbeschreibung, Systemanalyse und Experimente um Formate ergänzt, die ein systematisches Einbeziehen der relevanten Beteiligten bei Heliatek sicherstellte. Im Speziellen wurden mehrere interaktive Ziel- und Konzept-Workshops durchgeführt, deren Nutzen nicht nur für die eigentliche Simulationsstudie nachgewiesen werden konnte. Das Konzeptmodell wurde in einer an SysML angelehnten Darstellung realisiert - die Umsetzung des ausführbaren Modells erfolgte in Tecnomatix Plant Simulation.

Abbildung 1 stellt das Zusammenarbeitsmodell der Beteiligten bzgl. deren Mitwirkung in den entscheiden- den Phasen dar. Dieses Vorgehen wurde in Anlehnung an den partizipativen Planungsprozess nach Schenk et. al. gestaltet [10]. Im Kern zielt es darauf ab, möglichst frühzeitig die relevanten Beteiligten in partizipativen Formaten an der Ideenfindung und Lösungspräzisierung teilhaben zu lassen. Als Resultat lassen sich die Planungszeit reduzieren und Fehler vermeiden. In der Simulationsstudie hat sich der gezeigte Dreiklang aus Fachexperten, Simulationsexperten sowie aus wissenschaftlicher Unterstützung bewährt.

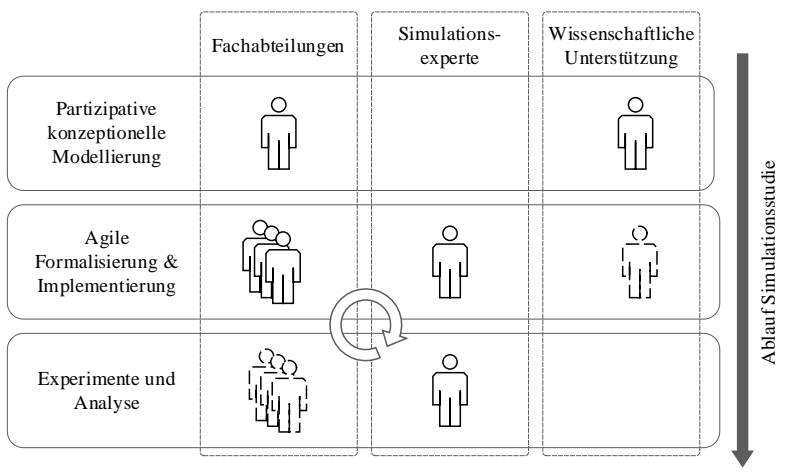

Figure 1: Partizipatives Simulationsvorgehen in Startups

Nachfolgend werden die zwei wesentlichen Phasen des Simulationsvorgehens beschrieben.

\section{PHASE 1: Partizipative top-down Konzeptmodeller- stellung}

Diese Phase startete mit der Aufgaben- und Zieldefinition der Simulationsstudie. Kern dieser waren interaktive Workshops, an welchem relevante Wissens- und Entscheidungsträger des Unternehmens eingebungen wurden. Dieser Tages-Workshop diente dem Zweck der Zielfindung und Aufgabendefinition für die Konzeptmodellerstellung.

Folgende inhaltliche Fragestellungen waren Bestandteil dieses ersten Schrittes der partizipativen Erarbeitung:

- Einführung in die Fertigungs- und Logistiksimulation sowie Schaffung eines simulativen Grundverständnisses unter Aufzeigen von Einsatzmöglichkeiten und Grenzen

- Gemeinsame Erarbeitung von Zielstellungen, die mit der Simulation verfolgt werden

- Einstieg in die Top-Down-Systemanalyse: Definition von Systemgrenzen und Systemelementen 
Mittels einer offenen und später moderierten Fragerunde in Abstimmung aller Beteiligten wurden potenzielle Zielstellungen gesammelt, priorisiert und kategorisiert. Als grundsätzliches Ergebnisziel konnte die Anordnung und Dimensionierung von Logistikmitteln, Lagerflächen sowie Personal im neu zu gestaltenden Fertigungsbereich (FAB2) identifiziert werden. Die weiteren Leistungsziele, welche für das Erreichen der Zielstellung nötig sind, wurden darin unterschieden, ob das Werkzeug der Simulation unmittelbar einen Mehrwert für diese Problemstellung bieten kann. Abbildung 2 stellt die im Zielworkshop erarbeiteten Ergebnis- und Leistungsziele der Simulationsstudie zusammen.

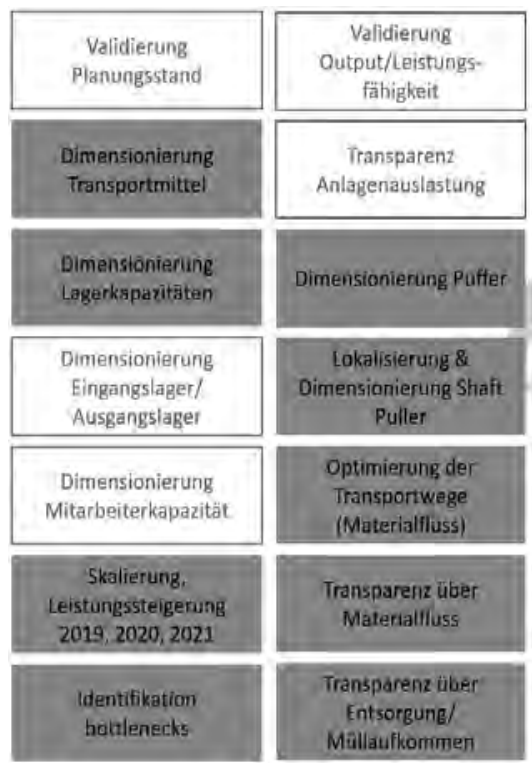

Figure 2: Ergebnis-/Leistungsziele der Studie

Ergänzend wurden weitere ,Randthemen` aufgenommen, welche durch das Unternehmen in den Planungsaktivitäten außerhalb dieses Projektes bearbeitet werden. Zusammenfassend wurde eine Entscheidungsunterstützung in folgenden Kategorien angestrebt:

- Transparenz der Fabrikabläufe auf Grundlage des aktuellen Planungsstandes

- Dimensionierung der Fertigungs- und Logistikmittel

- $\quad$ Anordnung ortsfester Fertigungsmittel

Aus den kollaborativ erarbeiteten Zielen leiteten sich die für die Erstellung des Konzeptmodells nötigen Eingangsdaten und Parameter ab. Weiterhin wurden die für die später durchzuführende Interpretation wichtigen
Messgrößen identifiziert. Auch hier zeigte sich, dass das frühzeitige Einbinden der Entscheidungsebene Missverständnisse und falsch eingesetzte Ressourcen vorbeugt.

Die mit der Leitungsebene im Rahmen des Zielworkshops abgestimmten Ergebnisse, wurden im nachfolgenden Detailworkshop mittels einer Systemanalyse vertieft. Der inhaltliche Input erfolgte durch Fachexperten aus den Bereichen Supply Chain Management, Ramp-Up, Produktionsplanung, Logistik sowie Fabrikintegration. Methodisch wurde der Workshop durch die Durchführenden der TU Chemnitz geleitet. Für die sich aus den Zielstellungen sowie den Vorarbeiten des Unternehmens ergebenden Objektklassen (Simulationselemente) wurden jeweils in Form von Expertengesprächen sequenziell folgende Inhalte erarbeitet

- Relevanz des Fabrikobjektes für die Simulation

- Festlegung des nötigen Abstraktionsgrades

- Definition nötiger Simulationsparameter sowie Inputs und Outputs

- Punktuelle Detaillierung des Ablaufverhaltens

In Theorie und Praxis hat es sich bewährt, das Konzeptmodell anhand folgender vier Schritte nach Möglichkeit sequenziell erarbeitet - auch der Detailworkshop lehnte sich an diese Schritte an:

- Hierarchie und Abgrenzung gegen die Systemumgebung

- Funktionale Analyse der Systemelemente

- Strukturelle Analyse der Systemelemente

- Analyse von Strategien und Prozessregeln

Aufgrund des nötigen Detaillierungsgrades bei gleichzeitig hoher Flexibilität wurde sich entschieden, die konzeptionelle und formale Modellierung in einem Modell zusammenzuführen. Für die Darstellung kam die grafische Modellierungssprache SysML zum Einsatz. Die darin bereitgestellten Diagramme eignen sich besonders für die Visualisierung komplexer Systeme. Damit kann die Aufbau- und auch die Ablaufstruktur modelliert und Zusammenhänge zwischen verschiedenen Darstellungen hergestellt werden. Für den ersten Teil des Zielworkshops wurden im Sinne der Schritte 1 und 2 sogenannte ,Blöcke‘ für feste und bewegliche Fabrikobjekte vorbereitet (Blockdiagramm). Mithilfe dieser wurden die relevanten Elemente der Simulation 
abgegrenzt sowie deren Funktion im Simulationsmodell detailliert in Abstimmung mit den Teilnehmern vertieft.

Die Ablaufstruktur stellt die Materialflussprozesse zwischen den Elementen eines Systems dar. Im Fall der Heliatek GmbH wurden die örtlich getrennten Fabrikräume und die Wege sowie Lager als Swimlanes abgebildet. Innerhalb dieser Swimlanes wurden die Lager und Betriebsmittel als Blöcke, die Materialflüsse, also die Logistikmittel und transportierten Elemente, als Pfeile dargestellt. Abbildung 3 zeigt einen Ausschnitt aus dem Aktivitätendiagramm.

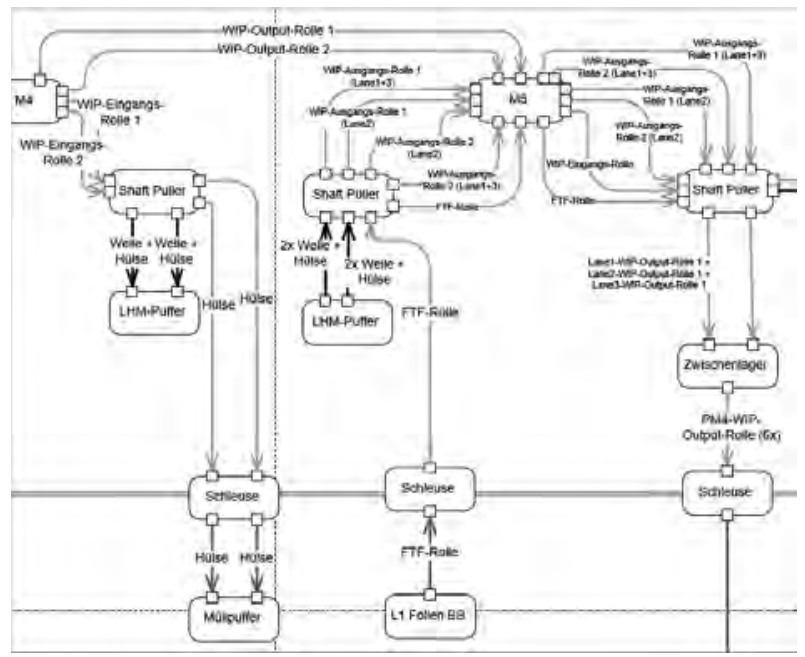

Figure 3: Ablaufstruktur modelliert als Aktivitätendiagramm

In der sich anschließenden Aufarbeitungsphase wurden die zusammengetragenen Informationen seitens der TU Chemnitz auf den Simulationsnutzen geprüft und auf dieser Basis das Konzeptmodell erarbeitet.

\section{PHASE 2: Agile Modellerstellung und Simulation}

Nach der Erarbeitung des Konzeptmodells sollte die in Planung befindliche Produktionslinie simulativ untersucht werden. Im Rahmen einer studentischen Abschlussarbeit wurde eine Materialflussanalyse durchgeführt.

Neben den in Phase 1 gezeigten Zielen, sollte weiterhin überprüft werden, inwieweit die Erwartungen und Zielstellungen der vorherigen Befragung im Unternehmen erfüllt wurden und welchen Mehrwert die Simulation im konkreten Fall bieten konnte.

Die Materialflusssimulation erfolgte vor der Fertigstel- lung der neuen Produktionsstrecke, um das Verhalten des Systems kennenzulernen und Rückschlüsse ziehen zu können, bevor es aufgebaut ist. Dies erfolgte in Vorbereitung des bevorstehenden Volumen-Ramp-up.

An die baulichen Bedingungen und Restriktionen am Standort in Dresden angepasst sowie aufgrund der vorherrschenden Produktstrukturen der einzelnen Produkte, wurde die Produktion auf zwei separate Hallenbereiche aufgeteilt. Sechs der insgesamt neun Produktionsmaschinen befinden sich im ersten Hallenteil. Hier findet die Basisfertigung statt. Die restlichen drei Maschinen stehen im nachgelagerten Hallenbereich, in welchem das Finishing der Rollen umgesetzt wird. Gefertigt werden Solarmodule im Rolle-zu-Rolle-Verfahren welche in Breite und Länge variabel sind. Es können Breiten zwischen $0,3 \mathrm{~m}$ und 1,2m sowie Längen zwischen $2 \mathrm{~m}$ und $12 \mathrm{~m}$ gefertigt werden.

Anknüpfend an das Konzeptmodells erfolgte anschließend die quantitative Datenbeschaffung nach Vorlage der im Konzeptmodell erarbeiteten Systemelemente. Die agile Datenbeschaffung gestaltete sich dermaßen, dass mit den jeweiligen Beteiligten die spezifischen Szenarien der Parameter erarbeitet und geplant wurden in Form von Einzelgesprächen. Zur einfachen DatenDokumentation erfolgte die Erhebung in Form von Excel-Sheets, um diese anschließend als Parameter in die Simulation zu überführen.

Im Konzeptmodell war unter anderem die Gesamtheit der Materialflüsse über die komplette Produktionsstrecke sowie den Lagerbereich erfasst. Dies diente als Grundlage zur Abbildung der Flüsse im Simulationsmodell. Zusätzlich wurden anhand von internen Planungsdokumenten die Rüst-, Bearbeitungs- sowie Wartungszeiten ermittelt und für das Modell aufbereitet. Weiterhin wurden Verfügbarkeiten pro Maschine in Abstimmung mit den Lieferanten ermittelt, da zum Zeitpunkt der Studie noch keine eigenen Erfahrungswerte dahingehend vorlagen.

Die Modellierung erfolgte anhand der zuvor generierten Daten und des Konzeptmodells.

Nach Fertigstellung des Modells wurden verschiedene Szenarien simuliert. Der Fokus lag hierbei auf der Untersuchung verschiedener Produkte und der Abhängigkeit von Maschinenauslastungen, Ausbringung und Durchlaufzeiten von den jeweiligen Produkten. Nach Rücksprache mit dem Unternehmen wurde sich im Rahmen der Simulationsversuche auf den ersten Hallen- 
teil fokussiert. Das komplette Modell wurde abschließend in einem Testszenario auf seine Funktionalität verifiziert. Im Weiteren werden nun die wichtigsten Ergebnisse der Simulationsversuche zum ersten Hallenteil in Abgleich mit den Erkenntnissen der vorangegangenen Befragungen erläutert.

Eine grundlegende Forderung war der allgemeine Erkenntnisgewinn über die Zusammenhänge des späteren Produktionssystems und die Generierung greifbarer Ergebnisse aus dem dynamischen Modell. Der Erkenntnisgewinn für das Unternehmen begann hierbei nicht erst bei der Durchführung und Auswertung der Versuche, sondern schon bei der agilen Datenaufnahme und deren statistischen Auswertung. Dies brachte bereits im Vorlauf der Versuche verwertbare Ergebnisse. So wurden für diverse Produkte die benötigten Bearbeitungszeiten ermittelt und erste Erkenntnisse über den Einfluss der Modullängen auf diese gewonnen. Die Maschine 2 ist mit dem Verdampfen der Organikmaterialien die maßgebende Anlage im Produktionsablauf. Ziel der Produktionsplanung ist es daher, diese Maschine möglichst auszulasten. Die Berechnungen haben ergeben, dass beim Fertigen kürzerer Module klare Tendenzen bestehen, dass andere Maschinen im Bottleneck liegen. Diese Feststellung wurde durch die Simulationsversuche untermauert. Ein Beispiel einer solchen Auswertung wird in der folgenden Abbildung gezeigt.

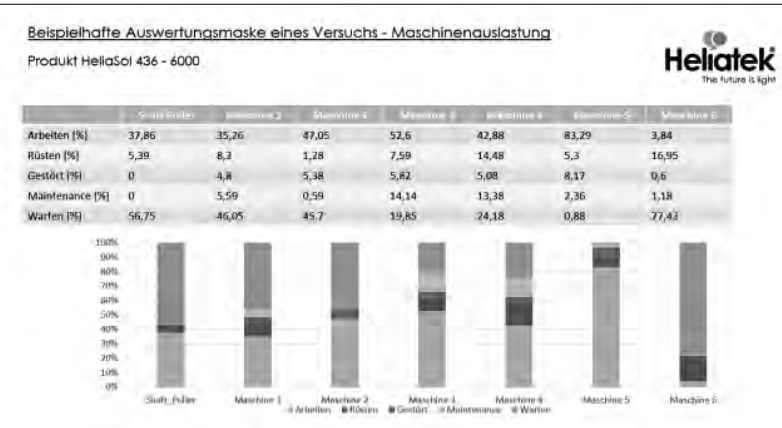

Figure 4: Produktspezifische Ergebnisauswertung

Für dieses Szenario ist die Maschine 5 die Anlage im Engpass und somit von besonderer Bedeutung bei der Planung und Steuerung der Produktionsprozesse.

Durch die Kombination der statischen Berechnungen und die nachfolgenden Simulationsversuche konnten die jeweiligen Maschinen im Bottleneck sowie die allgemeinen Auslastungen der Anlagen ermittelt werden.
Außerdem wurden die zu verarbeitenden Rollenlängen und Stückzahl je Produkt errechnet, welche nötig sind, um die jeweils gewünschte Maschine maximal auszulegen. Anschließend konnten diese Erkenntnisse durch Überprüfung mittels der Simulation bestätigt werden. So konnte die Simulation auch bei der Fragestellung nach der Identifizierung von Bottlenecks Antworten liefern. Dieses Vorgehen erfolgte in agilen Iterationsschleifen.

Von Interesse war außerdem die hinreichende Auslegung der Logistikmittel. So wurde deren relative Auslastung für die Versuche ausgewertet. Darüber hinaus wurden die Gabelstapler und der Shaft-Puller gesonderten Auswertungen unterzogen, um zu überprüfen, ob die derzeitige Planung hinsichtlich der Logistikmittel ausreichend ist. Der Shaft-Puller ist ein Hilfsmittel zum Handling der Hülsen, Wellen und kompletten Rollen und dient zum Vor- und Nachbereiten der Rollen.

Es hat sich gezeigt, dass die Stapler und der Shaft-Puller Tendenzen aufweisen, bei bestimmtem Systemkonstellationen zum Engpass werden. Hier bedarf es weiterer Untersuchungen, ob durch gezielte technische oder organisatorische Maßnahmen eine Beseitigung bzw. Verringerung dieser Tendenzen erreicht werden kann.

Neben den Anforderungen an die Studie wurden auch erwartete Grenzen und Probleme im Rahmen der Befragung aufgenommen. Hier wurde die Abbildung der komplexen Produktionsprozesse als mögliche Problemquelle beschrieben. In der Tat war die Realisierung der Prozesse mit den Mitteln in Plant Simulation eine der maßgeblichsten Herausforderungen beim Erstellen des Modells. Die Erstellung eines solchen Modells beansprucht einen nicht zu vernachlässigenden Zeitaufwand und ist für Start-ups und KMU ein zu berücksichtigender Faktor bei der Evaluierung, ob Simulation als Unterstützung infrage kommt. Eine weitere Problematik bei der vorliegenden Simulationsstudie lag in der fehlenden Datenbasis für die Inputangaben des Modells. Der Aufbau der Produktionsstrecke erfolgt in dieser Größenordnung erstmalig. Die installierten Anlagen sind allesamt Sonderanlagen, und so bestehen keine Erfahrungswerte hinsichtlich der Anlagentechnik und der Fertigungsprozesse. Viele Inputdaten beruhen daher auf Annahmen und sind im weiteren Verlauf stetig auf Richtigkeit zu überprüfen und zu aktualisieren. Hierbei haben die Szenariotechnik sowie das agile Vorgehen geholfen. 


\section{Handlungsempfehlungen für Start-Ups und KMU}

Zusammenfassend wird festgehalten, dass bezüglich der Chancen und Grenzen bei dem Einsatz von Simulation für Start-ups sowie kleine und mittlere Unternehmen noch weiterer Forschungsarbeit bedarf. Die Fachliteratur gibt nur begrenzt Aufschluss darüber, was Simulation konkret für diese Art von Unternehmen leisten kann und unter welchen Rahmenbedingungen ein Einsatz sinnvoll ist. Die größten Hindernisse, die einem breiten Einsatz von Simulationsanwendungen in den Unternehmen im Weg stehen, sind mit den hohen Anschaffungs- und Lizenzkosten sowie den zu komplexen Software-Tools und deren benötigtem Expertenwissen identifiziert worden. Gelingt es, die Rahmenbedingungen für einen Einsatz von Simulation für solche Unternehmen zu verbessern und Simulationsanwendungen zu entwickeln, welche auf die Bedürfnisse dieser ausgerichtet sind, kann Simulation eine entscheidende Unterstützung bei der Planung und Auslegung von Produktionsstätten für Start-ups und KMU sein.

Neben den allgemeinen Erkenntnissen kann für KMU zukünftige Handlungsempfehlungen abgeleitet werden:

- Die Gültigkeit der VDI 3633 kann auch in Start-ups/KMU bestätigt werden.

- $\quad$ Ein Top-Down-Vorgehen mit der Einbindung aller Entscheidungsebenen wird empfohlen.

- Aufgrund der oft heterogenen und dynamischen Datenlage, sind alle relevanten Wissensträger im Unternehmen möglichst früh in den Prozess einzubinden - dafür haben sich partizipative Methoden bewährt.

- Der Zweck sowie die Möglichkeiten und Grenzen der Simulation müssen häufig erst noch vermittelt werden - überambitionierte Zielstellungen sind zu vermeiden.

- Während der Modellierung ist mit vermehrten Änderungen in den Anforderungen und folglich im Modellaufbau zu rechnen. Aus diesem Grund bietet sich die agile, modulare Modellierung im ausführbaren Modell an.

- Das Simulationsmodell sollte als mitwachsender dauerhafter Begleiter agieren, mit dem ad hoc Simulation durchgeführt werden können (Erweiterung zum digitalen Zwilling sollte geprüft werden).
- In der Zusammenarbeit mit Hochschulen kann im Unternehmen systematisch SimulationKnowhow aufgebaut werden, was auch nach Beendigung der Studie Nutzen stiftet.

\section{Literaturverzeichnis}

[1] BRACHT, Uwe ; GECKLER, Dieter ; WENZEL, Sigrid: Digitale Fabrik : Methoden und Praxisbeispiele. 2., aktualisierte und erweiterte Auflage. Berlin : Springer Vieweg, 2018 (VDI-Buch)

[2] BISCHOFF, Jürgen ; TAPHORN, Christoph ; WOLTER, Denise ; Braun, Nomo ; FELLBAUM, Manfred ; Goloverov, Alexander ; LUDWIG, S. ; Hegmanns, T. ; Prasse, C. ; HenKe, M. ; OTHERS: Erschließen der Potenziale der Anwendung von Industrie 4.0 im Mittelstand. In: Berlin: Studie BMWi (2015)

[3] SPIECKERMANN, Sven: Durchgängige Planungsbegleitung mit Simulation im Mittelstand. In: ZWF Zeitschrift für wirtschaftlichen Fabrikbetrieb 103 (2008), 1-2, S. 83-85

[4] SCHUlZ, Andreas ; ZÜFLE, Edgar ; SOMMER, Lutz ; HAUG, Manuel: Simulation in der operativen Produktionsplanung - Erfolgsfaktoren für KMU. In: ZWF Zeitschrift für wirtschaftlichen Fabrikbetrieb 102 (2007), 1-2, S. 32-36

[5] SOMMER, Lutz; PlANKENHORN, Andreas: Simulation - Verborgene Chancen für den Mittelstand. In: ZWF Zeitschrift für wirtschaftlichen Fabrikbetrieb 99 (2004), Nr. 6, S. 303-305

[6] SCHUMACHER, Bastian Clemens: Beitrag zur Einarbeitung in ereignisdiskrete Simulation zur Neuund Umplanung von Materialflusssystemen. Berlin, Technische Universität Berlin, Fakultät V Verkehrs- und Maschinensysteme. Dissertation. 2019-12-12. URL https://dnb.info/1205804277/34

[7] WIMPFF, Daniel-Percy ; SEITZ, Stefan ; ERGIN, Tamer ; RoMmel, Steve ; HERMANN, Marco: simKMU - Internet- und webbasierte Simulationsdienste : Forschungsergebnisse im Teilvorhaben »Grundlagen, Konzeption und Intralogistik« des Verbundprojekts simKMU. Abschlussbericht. Stuttgart, 2011

[8] RudEL, Steffi: Prozess-Simulation in kleinen und mittleren Unternehmen mittels des Baukastensystems KMUSimMetall. In: WENZEL, Sigrid; PETER, Tim (Hrsg.): Simulation in Produktion und Logis- 
tik 2017 : kassel university press GmbH, 2017

[9] Technische Regel VDI 3633 Blatt 1:2014-12.

Simulation von Logistik-, Materialfluss- und Produktionssystemen - Grundlagen

[10] SCHENK, Michael ; WIRTH, Siegfried ; MÜLLER, Egon: Fabrikplanung und Fabrikbetrieb : Methoden für die wandlungsfähige, vernetzte und ressourceneffiziente Fabrik. 2., vollständig überarbeitete und erweiterte Auflage. Berlin, Heidelberg : Springer-Vieweg, 2014 (VDI-Buch) 